(c) American Dairy Science Association, 2003.

\title{
Reassessment of Biases in Predicted Nitrogen Flows to the Duodenum by NRC 2001
}

\section{N. R. St-Pierre}

Department of Animal Sciences

The Ohio State University, Columbus $43210^{1}$

\begin{abstract}
The appearance of numerous plots in recent literature from which the residuals are plotted against observed values $(\mathbf{Y})$ to assess a model's potential bias raises this question: should residuals be regressed against $\mathbf{Y}$ or against predicted values $(\hat{\mathbf{Y}})$ ? The answer requires knowing the expected relationship under the assumption of an unbiased model. The objectives of this paper are: 1) to derive the expected relationship between residuals, $\mathbf{Y}$, and $\hat{\mathbf{Y}} ; 2$ ) to determine whether $\mathbf{Y}$ or $\hat{\mathbf{Y}}$ should be used for the assessment of bias; and 3) to reassess the extent of mean and linear bias in the prediction of $\mathrm{N}$ flows to the duodenum by the NRC (2001). In the simplest case, we can assume a true model of the form $\mathbf{Y}=\mathbf{X} \boldsymbol{\beta}+\varepsilon$. This model is estimated by $\mathbf{Y}=\mathbf{X b}+\mathbf{e}$, and $\hat{\mathbf{Y}}=\mathbf{X b}$. The correlation between the residual vector $\mathbf{e}$ and the vector of observations $\mathbf{Y}$ can easily be derived. The numerator of the correlation coefficient is shown to be equal to $\mathbf{e}^{\prime} \mathbf{e}$, the residual sum of squares. The denominator of this correlation is equal to the square root of $\mathbf{e}^{\prime} \mathbf{e}$ multiplied by the total sum of squares. Algebraic simplifications show that the correlation between $\mathbf{e}$ and $\mathbf{Y}$ is equal to the square root of $\left(1-R^{2}\right)$. That is, under the assumption of an unbiased model, the residuals are correlated with the observed values and the slope of $\mathbf{e}$ regressed on $\mathbf{Y}$ is equal to $\left(1-R^{2}\right)$. Thus, a graph of $\mathbf{e}$ versus $\mathbf{Y}$ will show a positive slope between $\mathbf{e}$ and $\mathbf{Y}$ unless the model is a perfect predictor (i.e., $R^{2}$ is equal to 1.0). Significant slopes linking $\mathbf{e}$ to $\mathbf{Y}$ have been erroneously interpreted as evidence of biased models in the NRC (2001). Conversely, the slope of $\mathbf{e}$ regressed on $\hat{\mathbf{Y}}$ is expected to be zero under the assumption of an unbiased model. Therefore, residuals should be regressed against $\hat{\mathbf{Y}}$ and not $\mathbf{Y}$. When $\hat{\mathbf{Y}}$, as opposed to $\mathbf{Y}$, was used to assess biases in the prediction of flows to the duodenum of
\end{abstract}

\footnotetext{
Received June 17, 2002.

Accepted August 14, 2002

Corresponding author: N. R. St-Pierre; e-mail: st-pierre.8@osu.

${ }^{1}$ Salaries and research support were provided by state and federal funds appropriated to the Ohio Agricultural Research and Development Center, The Ohio State University. Manuscript No. 21-02AS.
} edu. microbial $\mathrm{N}$, nonammonia-nonmicrobial $\mathrm{N}$ and nonammonia N in NRC (2001), mean biases became nonsignificant and linear biases over the range of predicted values are of the same magnitude or smaller than the standard errors of measurements reported in literature. Thus, although $\mathrm{N}$ flow predictions from NRC (2001) may not be precise, they appear to have insignificant and inconsequential biases.

(Key words: prediction bias, model evaluation, National Research Council, accuracy)

Abbreviation key: $\mathbf{M N}=$ microbial nitrogen, $\mathbf{M S S}=$ model sum of squares, NANMN = nonammonia-nonmicrobial nitrogen, RSS = residual sum of squares, $\mathbf{T S S}=$ total sum of squares.

\section{INTRODUCTION}

The evaluation and validation of models, whether empirical or mechanical, require an assessment of prediction bias generally presented in the form of residual plots. Numerous residual plots have appeared in recent literature (NRC, 2001; Ruiz et al., 2001; Haig et al., 2002) from which residuals are plotted or regressed against observed values (Y) to assess linear bias even though statisticians (Draper and Smith, 1988) state that residuals should be plotted against predicted values $(\hat{\mathbf{Y}})$. It has long been known that residuals are correlated to observed values of the dependent variable in linear models. This is clearly stated in numerous statistical textbooks (e.g., Draper and Smith, 1988), although the proof of this relationship is not provided.

The NRC (2001) presents important plots of residuals against $\mathbf{Y}$ (Figures 5-6, 5-7, and 5-8) for the prediction of flows of three $\mathrm{N}$ pools to the duodenum. In most instances, there are clear negative slopes in these graphics, leading many to conclude that the NRC predictions have linear biases. The NRC (2001) stated that "the degree of the negative slope-bias that is evident in the residual plots are of concern" (NRC, 2001 p. 65). The report concluded that "errors in the structure of the model are probably major contributors to the negative slope biases" (NRC, 2001 p. 65). The objectives of this paper are 1) to show that a negative slope is expected when negative 
residuals are plotted against observed values if the predictions are truly unbiased, and 2) that the NRC predictions of $\mathrm{N}$ flows to the duodenum have small biases relative to measurement errors.

\section{MATERIALS AND METHODS}

\section{The Expected Relationship Between Residuals and Observed Values}

Let $Y_{i}$ be the $i^{\text {th }}$ observed value and $\hat{Y}_{i}$ be the $i^{\text {th }}$ predicted value. Residuals $e_{i}$ are defined as $e_{i}=Y_{i}-\hat{Y}_{i}$, that is the difference between the observed and the predicted value. The NRC (2001) and others defined the residuals $e_{i}^{*}=\hat{Y}_{i}-Y_{i}$. Thus $e_{i}=-e_{i}^{*}$ and the rest of this derivation could be expressed in terms of the $e_{i}^{*}$. The traditional expression of residuals in use in the field of statistics was chosen here. The expected relationship between $e_{i}$ and $Y_{i}$ under the conditions of an unbiased model must be established prior to making judgment that a certain pattern of residuals is symptomatic of biased predictions. That is, we must establish the expectation for the slope of the regression of $e_{i}$ on $Y_{i}$, and determine whether this expectation is equal to zero under unbiasedness. Likewise, we need to establish the expected value of $r_{\mathrm{eY}}$, the simple correlation coefficient between residuals and observed values, when the model is unbiased. As the following mathematical derivation will show, even in the simplest case, that of a simple linear model, the slope of the regression of $e_{i}$ on $Y_{i}$ and the correlation coefficient $r_{e Y}$ are not equal to zero. Similar conclusions can be reached for more complex model structures although the algebra gets very complex.

The correlation between $e_{i}$ and $Y_{i}$ is derived as follows. In the general case, the Pearson product-moment correlation $\mathrm{r}$ between two random variables $\mathrm{U}$ and $\mathrm{W}$ is given by:

$$
\mathbf{r}_{\mathrm{UW}}=\frac{\Sigma\left(\mathrm{U}_{\mathrm{i}}-\overline{\mathrm{U}}\right)\left(\mathrm{W}_{\mathrm{i}}-\overline{\mathrm{W}}\right)}{\left(\Sigma\left(\mathrm{U}_{\mathrm{i}}-\overline{\mathrm{U}}\right)^{2}\right)^{1 / 2}\left(\Sigma\left(\mathrm{W}_{\mathrm{i}}-\overline{\mathrm{W}}\right)^{2}\right)^{1 / 2}}
$$

where $\overline{\mathrm{U}}$ and $\overline{\mathrm{W}}$ are the means of the two random variables. Thus, the correlation between $\mathrm{e}_{\mathrm{i}}$ and $\mathrm{Y}_{\mathrm{i}}$ is given by:

$$
\mathrm{r}_{\mathrm{eY}}=\frac{\Sigma\left(\mathrm{e}_{i}-\overline{\mathrm{e}}\right)\left(\mathrm{Y}_{\mathrm{i}}-\overline{\mathrm{Y}}\right)}{\left(\Sigma\left(\mathrm{e}_{\mathrm{i}}-\overline{\mathrm{e}}\right)^{2}\right)^{1 / 2}\left(\Sigma\left(\mathrm{Y}_{\mathrm{i}}-\overline{\mathrm{Y}}\right)^{2}\right)^{1 / 2}}
$$

where $\overline{\mathrm{e}}$ and $\overline{\mathrm{Y}}$ are the means of residuals and of observed values respectively. A linear model is expressed as:

$$
\mathbf{Y}=\mathbf{X b}+\mathbf{e}
$$

where $\mathbf{Y}$ is a column vector of observed values, $\mathbf{X}$ is the matrix of independent variables, $\mathbf{b}$ is the vector of parameter estimates, and $\mathbf{e}$ is the column vector of residuals. The predicted values, $\hat{\mathbf{Y}}$, are calculated as:

$$
\hat{\mathbf{Y}}=\mathbf{X} \mathbf{b}
$$

The numerator in [2] can be simplified as follows:

$$
\Sigma\left(\mathrm{e}_{\mathrm{i}}-\overline{\mathrm{e}}\right)\left(\mathrm{Y}_{\mathrm{i}}-\overline{\mathrm{Y}}\right)=\Sigma \mathrm{e}_{\mathrm{i}}\left(\mathrm{Y}_{\mathrm{i}}-\overline{\mathrm{Y}}\right)
$$

because $\overline{\mathrm{e}}=0$ under the assumption of an unbiased model. That is, if the model is unbiased, then the mean residual (error) must be equal to zero. As we proceed with the multiplication of the factors in [5], the $\Sigma \mathbf{e}_{i} \bar{Y}$ part reduces to:

$$
\Sigma \mathrm{e}_{\mathrm{i}} \overline{\mathrm{Y}}=\mathrm{n} \overline{\mathrm{Y}} \Sigma \mathrm{e}_{\mathrm{i}}
$$

where $\mathrm{n}$ is the number of observations. Because $\overline{\mathrm{e}}=\Sigma \mathrm{e}_{\mathrm{i}} /$ $\mathrm{n}$ and $\overline{\mathrm{e}}=0$ under the assumption of an unbiased model, then $\Sigma \mathrm{e}_{\mathrm{i}}$ must also be equal to zero. Thus,

$$
\mathrm{n} \overline{\mathrm{Y}} \Sigma \mathrm{e}_{\mathrm{i}}=0
$$

Consequently, [5] reduces to

$$
\Sigma \mathrm{e}_{\mathrm{i}}\left(\mathrm{Y}_{\mathrm{i}}-\overline{\mathrm{Y}}\right)=\Sigma \mathrm{e}_{\mathrm{i}} \mathrm{Y}_{\mathrm{i}}
$$

In matrix notation,

$$
\mathbf{e}^{\prime} \mathbf{Y}=\Sigma \mathrm{e}_{\mathrm{i}} \mathrm{Y}_{\mathrm{i}}
$$

where $\mathbf{e}^{\prime}$ is the transpose (row vector) of the column vector e defined in [3]. Rearranging [3],

$$
\mathbf{e}=\mathbf{Y}-\mathbf{X b}
$$

The least squares estimates of $\mathbf{b}$ are unbiased and are found as:

$$
\mathbf{b}=\left(\mathbf{X}^{\prime} \mathbf{X}\right)^{-1} \mathbf{X}^{\prime} \mathbf{Y}
$$

where $\mathbf{X}^{\prime}$ is the transpose of $\mathbf{X}$, the design matrix defined in [3] and $\left(\mathbf{X}^{\prime} \mathbf{X}\right)^{-1}$ is the inverse of the $\mathbf{X}^{\prime} \mathbf{X}$ matrix. We define

$$
\mathbf{H}=\mathbf{X}\left(\mathbf{X}^{\prime} \mathbf{X}\right)^{-1} \mathbf{X}^{\prime}
$$

noting that $\mathbf{H}$ is symmetric and idempotent (i.e., $\mathbf{H}^{\prime} \mathbf{H}=$ H). Using [11] and [12], equation [10] becomes:

$$
\begin{gathered}
\mathbf{e}=\mathbf{Y}-\mathbf{X b} \\
\mathbf{e}=\mathbf{Y}-\mathbf{X}\left(\mathbf{X}^{\prime} \mathbf{X}\right)^{-1} \mathbf{X}^{\prime} \mathbf{Y}
\end{gathered}
$$




$$
\mathbf{e}=\mathbf{Y}-\mathbf{H Y}
$$

Factoring $\mathbf{Y}$ :

$$
\mathbf{e}=(\mathbf{I}-\mathbf{H}) \mathbf{Y}
$$

where $\mathbf{I}$ is the identity matrix, a symmetric and idempotent matrix.

Taking the transpose of [16]

$$
\mathbf{e}^{\prime}=\mathbf{Y}^{\prime}(\mathbf{I}-\mathbf{H})^{\prime}
$$

Using [16] and [17],

$$
\mathbf{e}^{\prime} \mathbf{e}=\mathbf{Y}^{\prime}(\mathbf{I}-\mathbf{H})^{\prime}(\mathbf{I}-\mathbf{H}) \mathbf{Y}
$$

Because both $\mathbf{I}$ and $\mathbf{H}$ are idempotent, $\mathbf{I}-\mathbf{H}$ is also idempotent and, by definition of an idempotent matrix,

$$
(\mathbf{I}-\mathbf{H})^{\prime}(\mathbf{I}-\mathbf{H})=(\mathbf{I}-\mathbf{H})
$$

Thus, [18] simplifies to

$$
\mathbf{e}^{\prime} \mathbf{e}=\mathbf{Y}^{\prime}(\mathbf{I}-\mathbf{H}) \mathbf{Y}
$$

and using [16]

$$
\mathbf{e}^{\prime} \mathbf{e}=\mathbf{Y}^{\prime} \mathbf{e}
$$

and

$$
\mathbf{e}^{\prime} \mathbf{e}=\mathbf{e}^{\prime} \mathbf{Y}
$$

due to the commutative property of vector multiplication. Combining [5], [8], [9], and [22]:

$$
\begin{gathered}
\Sigma\left(\mathrm{e}_{\mathrm{i}}-\overline{\mathrm{e}}\right)\left(\mathrm{Y}_{\mathrm{i}}-\overline{\mathrm{Y}}\right)=\Sigma \mathrm{e}_{\mathrm{i}}\left(\mathrm{Y}_{\mathrm{i}}-\overline{\mathrm{Y}}\right) \\
=\Sigma \mathrm{e}_{\mathrm{i}} \mathrm{Y}_{\mathrm{i}} \\
=\mathbf{e}^{\prime} \mathbf{Y} \\
=\mathbf{e}^{\prime} \mathbf{e}
\end{gathered}
$$

and $\mathbf{e}^{\prime} \mathbf{e}$ is known as the residual sum of squares (RSS). The denominator in [2] can be greatly simplified. The first part of the denominator reduces to:

$$
\Sigma\left(\mathrm{e}_{\mathrm{i}}-\overline{\mathrm{e}}\right)^{2}=\Sigma \mathrm{e}_{\mathrm{i}}^{2}
$$

because $\overline{\mathrm{e}}=0$ under the assumption that the model is unbiased. Using matrix notation.

$$
\Sigma \mathbf{e}_{\mathrm{i}}{ }^{2}=\mathbf{e}^{\prime} \mathbf{e}
$$

where $\mathbf{e}^{\prime} \mathbf{e}$ is again the RSS. The second half of the denominator in [2], $\Sigma\left(\mathrm{Y}_{\mathrm{i}}-\overline{\mathrm{Y}}\right)^{2}$ is, by definition, the total sum of squares (TSS). Finally, by definition, the model sum of squares (MSS) = TSS - RSS. Using [26], [27] and the definition of MSS, RSS, and TSS, [2] can be expressed as:

$$
\begin{aligned}
\mathrm{r}_{\mathrm{eY}} & =\frac{\mathrm{e}^{\prime} \mathrm{e}}{\left(\mathrm{e}^{\prime} \mathrm{e} \Sigma\left(\mathrm{Y}_{\mathrm{i}}-\overline{\mathrm{Y}}\right)^{2}\right)^{1 / 2}} \\
= & \frac{\mathrm{RSS}}{(\mathrm{RSS} \times \mathrm{TSS})^{1 / 2}} \\
= & \frac{\mathrm{RSS}}{\mathrm{RSS}^{1 / 2} \mathrm{TSS}^{1 / 2}} \\
& =\left(\frac{\mathrm{RSS}}{\mathrm{TSS}}\right)^{1 / 2}
\end{aligned}
$$

$$
=\left(\frac{\mathrm{TSS}-\mathrm{MSS}}{\mathrm{TSS}}\right)^{1 / 2}
$$

Because $\mathrm{R}^{2}=$ MSS/TSS, [33] becomes:

$$
\begin{gathered}
\mathrm{r}_{\mathrm{eY}}=\left(\frac{\mathrm{TSS}}{\mathrm{TSS}}-\mathrm{R}^{2}\right)^{1 / 2} \\
\mathrm{r}_{\mathrm{eY}}=\left(1-\mathrm{R}^{2}\right)^{1 / 2}
\end{gathered}
$$

Thus, under the assumption that the model is unbiased, a correlation is expected between the residuals and the observed values. This correlation is always positive and approaches 1 as $R^{2}$ approaches 0 . Even $R^{2}$ considered to be high would lead to significant $r_{\mathrm{eY}}$. For example, $\mathrm{r}_{\mathrm{eY}}=$ 0.32 when $R^{2}=0.9$.

The slope $\left(b_{1}\right)$ of the regression of $e_{i}$ on $Y_{i}$ is easily found:

$$
\mathrm{b}_{1}=\frac{\Sigma\left(\mathrm{e}_{\mathrm{i}}-\overline{\mathrm{e}}\right)\left(\mathrm{Y}_{\mathrm{i}}-\overline{\mathrm{Y}}\right)}{\Sigma\left(\mathrm{Y}_{\mathrm{i}}-\overline{\mathrm{Y}}\right)^{2}}
$$

Using [26] and the definitions of MSS, RSS and TSS, equation [36] becomes:

$$
\mathrm{b}_{1}=\frac{\mathrm{RSS}}{\mathrm{TSS}}
$$




$$
\begin{gathered}
\mathrm{b}_{1}=\frac{\mathrm{TSS}-\mathrm{MSS}}{\mathrm{TSS}} \\
\mathrm{b}_{\mathrm{i}}=1-\mathrm{R}^{2}
\end{gathered}
$$

This implies that for $\mathrm{R}^{2}<1$, there is a positive slope between $e_{i}$ and $Y_{i}$; and the lower the $R^{2}$, the greater the slope.

In [39], the slope is for the regression of $e_{i}$ on $Y_{i}$. Remember that NRC (2001) defined the residuals as $-\mathrm{e}_{\mathrm{i}}$. Thus, under the assumption of unbiased prediction, a negative slope would be expected between the NRC residuals and the observed values. The magnitude of this slope would be equal to $-\left(1-R^{2}\right)=R^{2}-1$. Clearly, biases of models predicting flows of nitrogenous compounds to the duodenum were not assessed properly. In nonlinear models with nonoptimized parameter estimates, the regression of $e_{i}$ on $Y_{i}$ can take a multitude of forms depending on the nature of the bias, if bias is present. The point is that the method of plotting (or regressing) residuals on observed values fails to properly identify biases with the simplest, most basic model (simple linear regression).

\section{The Expected Relationship Between Residuals and Predicted Values}

What can be said of the correlation of $e_{i}$ with the predicted values $\hat{Y}_{i}$ ? The numerator of the correlation $\mathrm{r}_{\mathrm{e}_{\mathrm{i}} \hat{\mathrm{Y}}}$ is:

$$
\begin{gathered}
\text { Numerator of } \mathrm{r}_{\mathrm{e}_{\mathrm{i}} \hat{\mathrm{Y}}}=\Sigma\left(\mathrm{e}_{\mathrm{i}}-\overline{\mathrm{e}}\right)\left(\hat{\mathrm{Y}}_{\mathrm{i}}-\overline{\hat{\mathrm{Y}}}\right) \\
=\Sigma \mathrm{e}_{\mathrm{i}}\left(\hat{\mathrm{Y}}_{\mathrm{i}}-\overline{\hat{\mathrm{Y}}}\right)
\end{gathered}
$$

because $\overline{\mathrm{e}}=0$ under the assumption of an unbiased model. Factoring,

$$
\begin{gathered}
\text { Numerator of } \mathrm{r}_{\mathrm{e}_{\mathrm{i}} \hat{\mathrm{Y}}}=\Sigma \mathrm{e}_{\mathrm{i}} \hat{\mathrm{Y}}_{\mathrm{i}}-\Sigma \mathrm{e}_{\mathrm{i}} \overline{\hat{Y}}_{\mathrm{i}} \\
=\Sigma \mathrm{e}_{\mathrm{i}} \hat{\mathrm{Y}}_{\mathrm{i}}
\end{gathered}
$$

because $\Sigma \mathrm{e}_{\mathrm{i}}=0$ under the assumption of an unbiased model. This is because of $\overline{\mathrm{e}}=0$ and that $\overline{\mathrm{e}}=\Sigma \mathrm{e}_{\mathrm{i}} \div \mathrm{n}$. In matrix notation,

$$
\Sigma \mathbf{e}_{\mathrm{i}} \hat{\mathbf{Y}}_{\mathrm{i}}=\mathbf{e}^{\prime} \hat{\mathbf{Y}}
$$

We know from [4] that

$$
\hat{\mathbf{Y}}=\mathbf{X} \mathbf{b}
$$

And from [11] that

$$
\mathbf{b}=\left(\mathbf{X}^{\prime} \mathbf{X}\right)^{-1} \mathbf{X}^{\prime} \mathbf{Y}
$$

Thus:

$$
\begin{gathered}
\hat{\mathbf{Y}}=\mathbf{X}\left(\mathbf{X}^{\prime} \mathbf{X}\right)^{-1} \mathbf{X}^{\prime} \mathbf{Y} \\
\hat{\mathbf{Y}}=\mathbf{H} \mathbf{Y}
\end{gathered}
$$

Also, from [16]

$$
\mathbf{e}=(\mathbf{I}-\mathbf{H}) \mathbf{Y}
$$

and from [17]

$$
\mathbf{e}^{\prime}=\mathbf{Y}^{\prime}(\mathbf{I}-\mathbf{H})^{\prime}
$$

So, using [48] and [50],

$$
\begin{array}{r}
\mathbf{e}^{\prime} \hat{\mathbf{Y}}=\mathbf{Y}^{\prime}(\mathbf{I}-\mathbf{H})^{\prime} \mathbf{H Y} \\
\mathbf{e}^{\prime} \hat{\mathbf{Y}}=\mathbf{Y}^{\prime}\left(\mathbf{I}^{\prime}-\mathbf{H}^{\prime}\right) \mathbf{H Y}
\end{array}
$$

Factoring,

$$
\mathbf{e}^{\prime} \hat{\mathbf{Y}}=\mathbf{Y}^{\prime}\left(\mathbf{I}^{\prime} \mathbf{H}-\mathbf{H}^{\prime} \mathbf{H}\right) \mathbf{Y}
$$

Because $\mathbf{H}$ is idempotent and $\mathbf{I}$ is the identity matrix,

$$
\mathbf{e}^{\prime} \hat{\mathbf{Y}}=\mathbf{Y}^{\prime}(\mathbf{H}-\mathbf{H}) \mathbf{Y}
$$

Which results in:

$$
\mathbf{e}^{\prime} \hat{\mathbf{Y}}=0
$$

Thus, the numerator of $r_{e_{i}}$ is zero and the correlation between $e_{i}$ and $\hat{Y}_{i}$ is zero. That is, the residuals are not correlated with the predictions, and the slope of $e_{i}$ regressed on $\hat{Y}_{i}$ is zero if the model is unbiased. A positive or negative slope of $e_{i}$ on $\hat{Y}_{i}$ is a test of biased prediction. This is why we should plot the residuals versus the predicted values and not the observed values.

\section{Reevaluation of Biases in NRC Predictions of N Flows}

Data in figures 5-6, 5-7 and 5-8 of NRC (2001) were digitized using a GTCO CalComp AccuTabII digitizer (Columbia, MD) set at a resolution of $0.05 \mathrm{~mm}$, to recalculate observed, predicted and residual values for microbial nitrogen (MN), nonammonia-nonmicrobial nitrogen (NANMN), and nonammonia nitrogen (NAN) flows to the duodenum. For all three $\mathrm{N}$ flow variables, residuals were regressed against their predicted values according to the following model (expressed here for MN): 
Table 1. Descriptive statistics of observed, predicted and residuals values of microbial N, nonammonianonmicrobial N (NANMN), and nonammonia N (NAN) flows to the duodenum. ${ }^{1}$

\begin{tabular}{lcrrrr}
\hline Variable & $\mathrm{n}$ & Mean & SD & Min. & Max. \\
\hline Microbial N (g/d) & & & & & \\
Observed & 284 & 244.9 & 77.2 & 85.5 & 487.1 \\
Predicted & 284 & 246.4 & 50.2 & 119.3 & 351.9 \\
Residual & 284 & -1.4 & 66.6 & -137.6 & 236.0 \\
Nonammonia-nonmicrobial N (g/d) & & & & \\
Observed & 275 & 210.7 & 89.9 & 33.7 & 431.4 \\
Predicted & 275 & 210.5 & 71.7 & 58.1 & 373.7 \\
Residual & 275 & 0.2 & 65.5 & -203.5 & 187.5 \\
Nonammonia N (g/d) & & & & & \\
Observed & 275 & 455.1 & 130.1 & 94.9 & 856.3 \\
Predicted & 275 & 464.0 & 118.8 & 136.4 & 713.6 \\
Residual & 275 & -8.9 & 85.7 & -262.2 & 217.9 \\
\hline
\end{tabular}

${ }^{1}$ Data are from NRC (2001) figures 5-6, 5-7 and 5-8. Data were originally published in 99 studies listed in table 5-8 of NRC (2001).

$$
\mathrm{e}_{\mathrm{i}}=\mathrm{b}_{0}+\mathrm{b}_{1}\left(\mathrm{MN}_{\mathrm{i}}-\overline{\mathrm{MN}}\right)+\check{\mathrm{e}}_{\mathrm{i}}
$$

where

$\mathrm{e}_{\mathrm{i}}$ is the $\mathrm{i}^{\text {th }}$ residual value,

$b_{0}, b_{1}$ are parameter estimates (fixed effects),

$\mathrm{MN}_{\mathrm{i}}$ is the $\mathrm{i}^{\text {th }}$ predicted value of $\mathrm{MN}$,

$\overline{\mathrm{MN}}$ is the mean of all predicted values of $\mathrm{MN}$, and

$\check{\mathrm{e}}_{\mathrm{i}}$ is the error of the regression of residuals on predicted values.

In [55], the regressor is shifted and centralized to its mean value. This method of shifting the independent variable was extensively used by Harvey $(1977 ; 1982)$ in the software LSML76 to test the interaction of a discrete (class) variable by a continuous variable. By centering the independent variable, the intercept term of the linear model is estimated at the mean value of the independent variable as opposed to a value of zero. The intercept term at the mean value of the regressor measures the overall prediction bias, also known as mean prediction bias. A t-test on the estimate of the intercept determines the statistical significance of this bias. The slope of the regression is an estimate of the linear prediction bias and a t-test is used to assess its significance. In instances where the slope of the regression of residuals on predicted values is significant, the magnitude of the bias within the range of the predicted values must be quantified. This is done by calculating the bias at the minimum and maximum levels of the predicted values. Equation [55] is used with the minimum and maximum predicted values as inputs. Then, the bias at minimum and maximum predictions is judged relative to the size of the standard error $\left(\sigma_{\text {ĕi }}\right)$, or compared to the $95 \%$ confidence intervals of measurements reported in the literature.

\section{RESULTS AND DISCUSSION}

Table 1 reports summary statistics regarding observed, predicted and residual values for MN, NANMN and NAN that are presented in figures 5-6, 5-7 and 5-8 of NRC (2001).

\section{Microbial N Flow to the Duodenum}

Figure 1 shows the relationship between residuals $\mathrm{MN}$ (i.e., observed MN minus predicted MN) and observed $\mathrm{MN}$. The slope of the regression of residuals on observed $\mathrm{MN}$ is $0.66( \pm 0.04)$. From [39], one would expect this slope to equal $\left(1-R^{2}\right)$ if the predictions are unbiased. The actual $\mathrm{R}^{2}$ (the square of the simple Pearson correlation coefficient between observed and predicted values) is 0.28 . Thus the slope of the regression of residuals on observed values would be $1-0.28=0.72$ under the

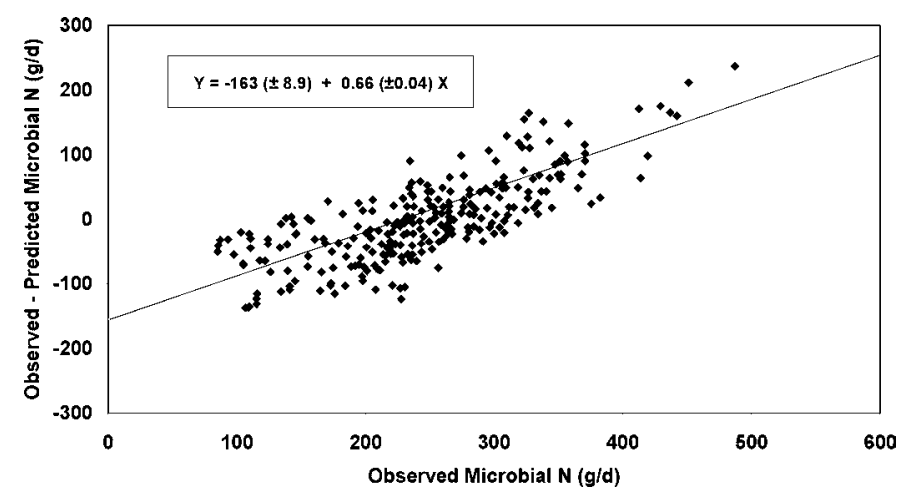

Figure 1. Plot of observed minus predicted microbial $\mathrm{N}$ flow to the duodenum (MN) vs. observed MN. Data are from NRC (2001) figure 5-6. Both the intercept and the slope are significantly different from $0(P<0.01)$, leading to the erroneous conclusion that the model generating the prediction is biased. 


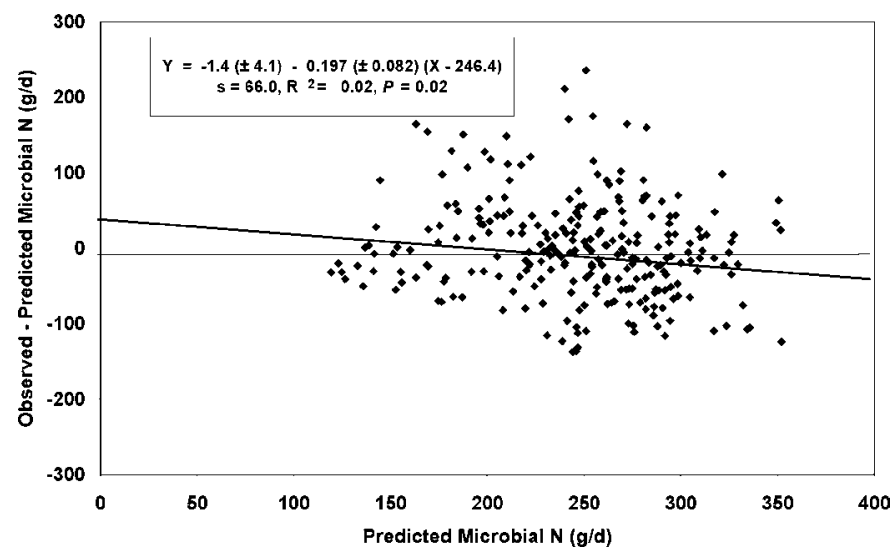

Figure 2. Plot of observed minus predicted microbial $\mathrm{N}$ flow to the duodenum (MN) vs. predicted MN. Data are from NRC (2001) figure 5-6. The independent variable predicted MN was centered around the mean predicted value before the residuals were regressed on the predicted values. The mean bias $(-1.4 \mathrm{~g} / \mathrm{d})$ is not significant. The linear bias $(0.197)$, although significant statistically $(P=0.02)$ results in a maximum bias of less than $27 \mathrm{~g} / \mathrm{d}$ over the full range of predicted values.

assumption of an unbiased model. The difference between 0.66 and 0.72 is due to (1) the nonlinear prediction model used by NRC, (2) random variance in estimation, and, (3) because the model is not optimized with respect to the residual values (i.e., the parameters used in the NRC model are not optimized with respect to the residual values-they are not least-squares estimates). The similarity of the slope and $\left(1-\mathrm{R}^{2}\right)$ should be taken as evidence of an unbiased model.

Figure 2 shows the relationship between residual MN and predicted MN, with the predicted values shifted so as to be centered at the mean predicted MN of $246 \mathrm{~g} / \mathrm{d}$.

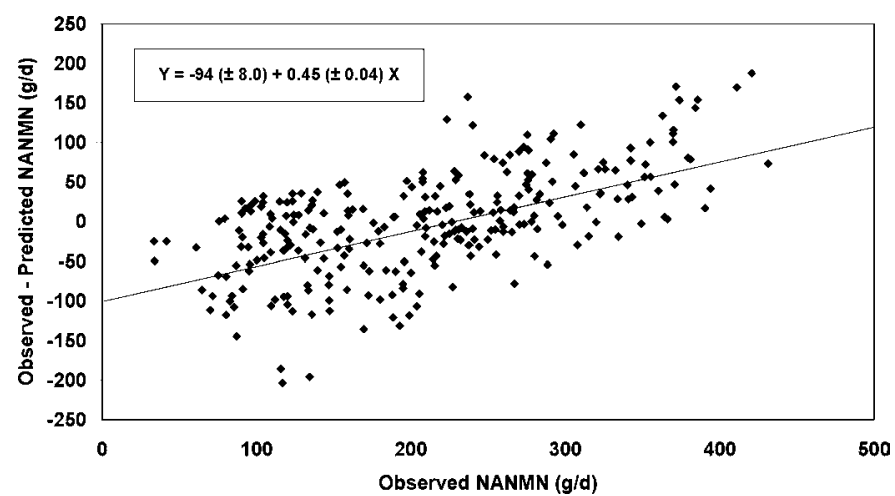

Figure 3. Plot of observed minus predicted nonammonia-nonmicrobial $\mathrm{N}$ flow to the duodenum (NANMN) vs. observed NANMN. Data are from NRC (2001) figure 5-7. Both the intercept and the slope are significantly different from $0(P<0.01)$, leading to the erroneous conclusion that the model generating the prediction is biased.

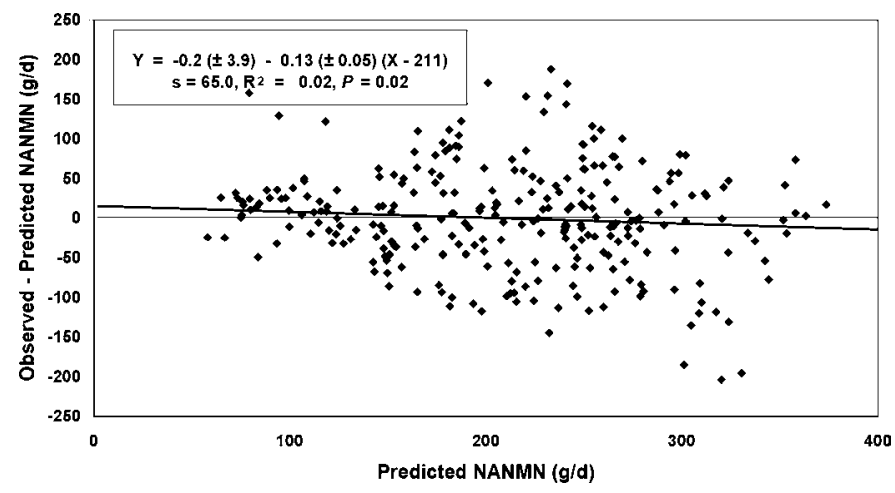

Figure 4. Plot of observed minus predicted nonammonia-nonmicrobial $\mathrm{N}$ flow to the duodenum (NANMN) vs. predicted NANMN. Data are from NRC (2001) figure 5-7. The independent variable predicted NANMNMN was centered around the mean predicted value before the residuals were regressed on the predicted values. The mean bias $(-0.2 \mathrm{~g} / \mathrm{d})$ is not significant. The linear bias $(-0.13)$, although significant statistically $(P=0.02)$ results in a maximum bias of less than $21 \mathrm{~g} / \mathrm{d}$ over the full range of predicted values.

That is, the independent variable is predicted $\mathrm{MN}$ minus the mean predicted MN. As explained previously, this procedure results in an exact test for the mean bias. By shifting the independent variable, the two parameter estimates (slope and intercept) are orthogonal and, consequently, independent of each other. Thus, a test on the location of the intercept is independent from the slope estimate. Results showed an absence of mean bias $(P=0.73)$, but a significant linear bias $(P=0.02)$. The magnitude of this linear bias translates, however, to values of less than $27 \mathrm{~g} / \mathrm{d}$ at the minimum $(119 \mathrm{~g} / \mathrm{d})$ and $22 \mathrm{~g} / \mathrm{d}$ at the maximum $(352 \mathrm{~g} / \mathrm{d})$ predicted $\mathrm{MN}$ values. These are less than the standard error ( $66 \mathrm{~g} / \mathrm{d})$, and well within the $95 \%$ confidence interval of least-square means reported in the literature for MN measurements (Aldrich

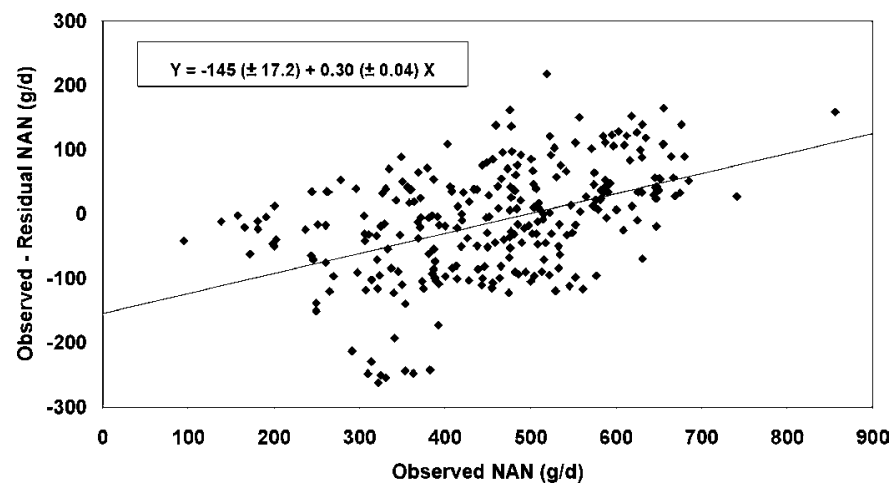

Figure 5. Plot of observed minus predicted nonammonia $\mathrm{N}$ flow to the duodenum (NAN) vs. observed NAN. Data are from NRC (2001) figure 5-8. Both the intercept and the slope are significantly different from $0(P<0.01)$, leading to the erroneous conclusion that the model generating the prediction is biased. 


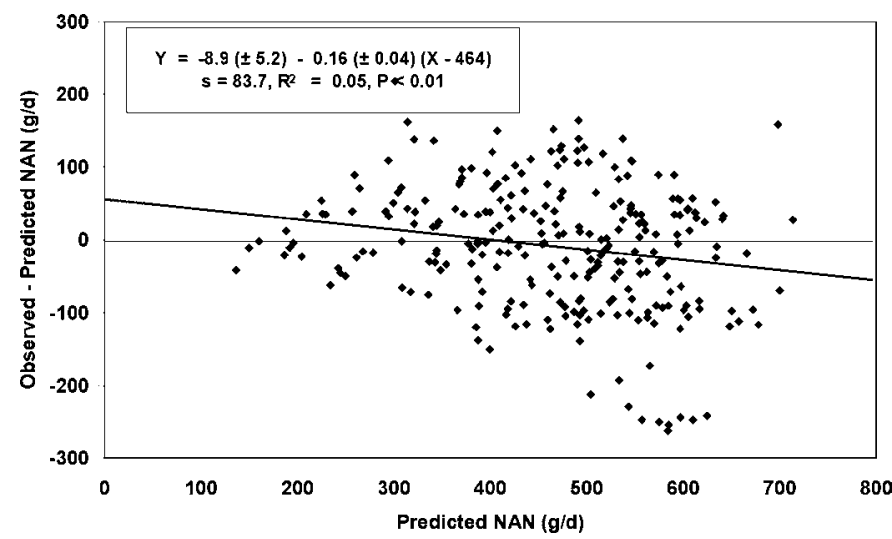

Figure 6. Plot of observed minus predicted nonammonia $\mathrm{N}$ flow to the duodenum (NAN) vs. predicted NAN. Data are from NRC (2001) figure 5-8. The independent variable predicted MN was centered around the mean predicted value before the residuals were regressed on the predicted values. The mean bias $(-9 \mathrm{~g} / \mathrm{d})$ is not significant. The linear bias $(-0.16)$, although significant statistically $(P<0.01)$ results in a maximum bias of less than $49 \mathrm{~g} / \mathrm{d}$ over the full range of predicted values.

et al., 1993; Cunningham et al., 1994; Beauchemin et al., 1999).

\section{Nonammonia-Nonmicrobial N Flow to the Duodenum}

The relationship between residual NANMN and observed NANMN is shown in Figure 3. The slope of the regression of residuals on observed NANMN is 0.45 ( \pm $0.04)$. Again, this value is close to $\left(1-R^{2}\right)$ where $R^{2}$ equals 0.48 . The regression of residuals on predicted NANMN values is shown in Figure 4 . The mean bias is clearly nonsignificant (intercept $=0.2 \pm 3.9, P=0.97$ ) whereas the linear bias (slope $=-0.131 \pm 0.05, P=0.02$ ) although significant, implies a bias of less than $20 \mathrm{~g} / \mathrm{d}$ at the minimum $(58.1 \mathrm{~g} / \mathrm{d})$ and of less than $21 \mathrm{~g} / \mathrm{d}$ at the maximum $(373.7 \mathrm{~g} / \mathrm{d})$ predicted NANMN values. These biases are less than the standard error $(65 \mathrm{~g} / \mathrm{d})$ and within the 95\% confidence range of measurements found in the literature (McCarthy et al., 1989; Cunningham et al., 1994).

\section{Nonammonia N Flow to the Duodenum}

Figure 5 shows the relationship between residual NAN and observed NAN. The slope of the regression of residuals on observed NAN is $0.30( \pm 0.04)$ compared to ( 1 $\left.-\mathrm{R}^{2}\right)=0.41$. The regression of residual NAN on predicted NAN is shown in Figure 6. The mean bias is nonsignificant (intercept $=-8.9 \pm 5.2, P=0.09$ ) whereas the linear bias (slope $=-0.161 \pm 0.044, P<0.01$ ) implies a bias of less than $44 \mathrm{~g} / \mathrm{d}$ at the minimum $(136.4 \mathrm{~g} / \mathrm{d})$ and of less than $49 \mathrm{~g} / \mathrm{d}$ at the maximum $(713.6 \mathrm{~g} / \mathrm{d})$ predicted NAN values. These biases are smaller than the standard error $(83.7 \mathrm{~g} / \mathrm{d})$ and within the $95 \%$ confidence range of the measured values reported in the literature (McCarthy et al., 1989; Aldrich et al., 1993; Cunningham et al., 1994).

\section{CONCLUSIONS}

Residual plots for the detection of mean and linear model biases should be based on predicted values for the $\mathrm{X}$-axis. Under the assumption of an unbiased model, the slope of the regression between the residuals and the observed values is expected to be equal to $1-R^{2}$. A proper analysis of the residual values reported by NRC (2001) for MN, NANMN and NAN failed to detect any mean bias of prediction for the NRC model. Linear biases were in all instances small and of lesser magnitude over the full range of predicted values than reported errors of measurements. Thus, predictions of $\mathrm{N}$ flows to the duodenum using the NRC (2001) model appear to have small biases relative to the error of measurements. It is likely that future improvements in prediction properties will only be gained if methods of measurements and experimental designs are improved to yield much smaller errors of measurements.

\section{REFERENCES}

Aldrich, J. M., L. D. Muller, G. A. Varga, and L. C. Griel Jr. 1993. Nonstructural carbohydrate and protein effects on rumen fermentation, nutrient flow, and performance of dairy cows. J. Dairy Sci. 76:1091-1105.

Beauchemin, K. A., W. Z. Yang, and L. M. Rode. 1999. Effects of grain source and enzyme additive on site and extent of nutrient digestion in dairy cows. J. Dairy Sci. 82:378-390.

Cunningham, K. D., M. J. Cecava, and T. R. Johnson. 1994. Flow of nitrogen and amino acids in dairy cows fed diets containing supplemental feather meal and blood meal. J. Dairy Sci. 77:3666-3675.

Draper, N. R., and H. Smith. 1988. Pages 63-64 in Applied Regression Analysis. 3rd ed. John Wiley and Sons, Inc., New York, NY.

Haig, P. A., T. Mutsvangwa, R. Spratt, and B. W. McBride. 2002. Effects of dietary protein solubility on nitrogen losses from lactating dairy cows and comparison with predictions from the Cornell Net Carbohydrate and Protein System. J. Dairy Sci. 85:1208-1217.

Harvey, W. R. 1976. User's Guide for LSML76. The Ohio State University, Columbus.

Harvey, W. R. 1982. Mixed model capabilities of LSML76. J. Anim. Sci. 54:1279-1285.

McCarthy, R. D. Jr., T. H. Klusmeyer, J. L. Vicini, J. H. Clark, and D. R. Nelson. 1989. Effects of source of protein and carbohydrate on ruminal fermentation and passage of nutrients to the small intestine of lactating cows. J. Dairy Sci. 72:2002-2016.

National Research Council. 2001. Pages 65-67 in Nutrient Requirements of Dairy Cattle. 7th rev. ed. Natl. Acad. Sci., Washington, DC.

Ruiz, R., G. L. Albrecht, L. O. Tedeshi, G. Jarvis, J. B. Russell, and D. G. Fox. 2001. Effect of monensin on the performance and nitrogen utilization of lactating dairy cows consuming fresh forage. J. Dairy Sci. 84:1717-1727. 